\title{
KH176 under development for rare mitochondrial disease: a first in man randomized controlled clinical trial in healthy male volunteers
}

Saskia Koene ${ }^{1}$, Edwin Spaans², Luc Van Bortel ${ }^{3}$, Griet Van Lancker ${ }^{3}$, Brant Delafontaine ${ }^{3}$, Fabio Badilini ${ }^{4}$, Julien Beyrath ${ }^{2}$ and Jan Smeitink, ${ }^{1,2}$

\begin{abstract}
Background: Mitochondrial disorders are a clinically, biochemically and genetically heterogeneous group of multisystem diseases, with an unmet medical need for treatment. $\mathrm{KH} 176$ is an orally bio-available small molecule under development for the treatment of mitochondrial(-related) diseases. The compound is a member of a new class of drugs, acting as a potent intracellular redox-modulating agent essential for the control of oxidative and redox pathologies. The aim of this randomized, placebo controlled, double-blinded phase 1 study was to test safety, tolerability and pharmacokinetics of single and multiple doses of $\mathrm{KH} 176$ in healthy male volunteers. Putative effects on redox related biomarkers were explored.
\end{abstract}

Results: KH176 was well tolerated up to and including a single dose of $800 \mathrm{mg}$ and multiple doses of $400 \mathrm{mg}$ b.i.d. for 7 Days. However, when the QT interval was corrected for heart rate, administration of single doses of 800 and $2000 \mathrm{mg}$ and at a multiple dose of $400 \mathrm{mg} \mathrm{KH176}$ had marked effects. Post-hoc analysis of the ECGs showed clear changes in cardiac electrophysiology at single doses of 800 and $2000 \mathrm{mg}$ and multiple doses of $400 \mathrm{mg}$ b.i.d.. At lower doses, detailed ECG analysis showed no changes in electrophysiology compared to placebo. Exposure-response modelling of the cardiac intervals revealed an exposure range of $\mathrm{KH} 176$ without effects on cardiac conduction and provided a threshold of $1000 \mathrm{ng} / \mathrm{mL}$ above which changes in intervals could occur. After single- and multiple-dose administration, the pharmacokinetics of $\mathrm{KH} 176$ was more than dose proportional. KH176 accumulated to a small extent and food only slightly affected the pharmacokinetics of KH176, which was considered clinically irrelevant. Renal excretion of unchanged $\mathrm{KH} 176$ and its metabolite represents a minor pathway in the elimination of $\mathrm{KH} 176$. As expected in healthy volunteers no effects on redox biomarkers were observed.

Conclusion: The study deemed that KH176 is well tolerated up to single doses of $800 \mathrm{mg}$ and multiple doses of $400 \mathrm{mg}$ b.i.d. and has a pharmacokinetic profile supportive for a twice daily dosing. Only at high doses, KH176 causes clinically relevant changes in cardiac electrophysiology, including prolonged QTc interval and changes in T wave morphology. A Phase 2 clinical trial (100 mg b.i.d., orally) has been conducted recently of which the final results are expected Q1 2018.

Trial registration: NCT02544217. Registered. ISRCTN43372293. Retrospectively registered.

Keywords: Randomized controlled trial, Mitochondrial medicine, Clinical trial phase 1, Mitochondrial disorder, Orphan drugs, Rare disease, KH176, Redox, Pharmacokinetics, Safety

\footnotetext{
* Correspondence: info@khondrion.com

${ }^{1}$ Radboud Center for Mitochondrial Medicine (RCMM) at the Department of

Pediatrics, Radboud university medical center, Geert Grooteplein Zuid 10, PO BOX 9101, 6500, HB, Nijmegen, The Netherlands

${ }^{2}$ Khondrion BV, Philips van Leydenlaan 15 (427), 6525, EX, Nijmegen, The

Netherlands

Full list of author information is available at the end of the article
} 


\section{Background}

Over 1150 genes have been identified to encode for proteins located in the mitochondria [1]. Mutations in these genes, like those involved in oxidative phosphorylation, can cause mitochondrial disease. This genetic heterogeneity is one of the numerous factors explaining the phenotypic variability seen in mitochondrial diseases, including many mono-, and multisystem phenotypes in childhood and adulthood [2]. Although the rate of deterioration is variable, the disease course is often progressive and the prognosis of some of the childhood diseases is even very poor [3]. With a minimal prevalence rate of about 1 in 4300-5000 [4], there is a clear need for therapy for these devastating disorders.

Currently, there is no effective treatment for most mitochondrial disorders and the care for these patients is mainly supportive [4]. Many treatment strategies have been attempted, including gene therapy, exercise and nutritional therapy, modulation of cell signalling and the manipulation of reactive oxygen species [5-7]. The latter has been subject of many studies and clinical trials $[8,9]$, since it has been recognised that cell redox imbalance play a key role in the pathogenesis of many of the clinical manifestations of mitochondrial diseases [5, 10-12].

$\mathrm{KH} 176$ (and its active metabolite $\mathrm{KH} 176 \mathrm{~m}$ ) is a potent intracellular redox-modulating agent targeting these reactive oxygen species. Preclinical studies in patient-derived fibroblasts with a wide variety of gene defects associated with mitochondrial dysfunction show that KH176 was able to protect these cells against a toxic perturbation of their redox balance. Additionally, a 4 week- in vivo study showed that KH176 was able to significantly improve motor performance and gait in Ndufs $4^{-/-}$mice [13]. Translating preclinical results to an anticipated human dose indicates that $100 \mathrm{mg}$ b.i.d. is expected to be within a putatively efficacious range.

This study aims to test the safety, tolerability and pharmacokinetics of KH176 in human subjects after oral administration of a single dose (SD) as well as multiple doses (MD), to obtain a safe and putatively efficacious dosing regimen of KH176 for a next Proof of Concept study.

\section{Methods}

The compound as described herein was tested in a double-blinded, randomized, placebo-controlled, singlecentre study in healthy male volunteers (Fig. 1). The single ascending dose (SAD) part has a partial alternating crossover design $(n=6)$ and the multiple-ascending dose (MAD) part has a sequential group design. Randomization was 2:1 (2 active for each placebo). All subjects received placebo once.

\section{Study population}

For both the SAD and MAD study, healthy men between 18 and 55 years of age with a body mass index (BMI) of $18.0-30.0 \mathrm{~kg} / \mathrm{m}^{2}$ were recruited. Good physical and mental health was established by medical history, physical examination, electrocardiogram (ECG) and vital signs recording, and results of clinical chemistry, hematology and urinalysis testing within 4 weeks prior to the first dose. Participants agreed to stay in the clinic during the first $24 \mathrm{~h}$ after dosing (SAD) and during Day 8 (MAD) and refrain from multivitamins and dietary supplements and grapefruit juice at least 14 Days prior to the first dosing, from alcohol 7 Days prior to the first dosing, from strenuous exercise, beverages containing quinine and from xanthine-derivates (e.g. caffeine) $48 \mathrm{~h}$ prior to the clinical admission and during the study. Only non-smokers (at least 3 months) were eligible for inclusion. Exclusion criteria included: clinically significant allergies, positive serology for hepatitis B surface antigen, hepatitis $\mathrm{C}$ antibodies, HIV1 of HIV2, history of alcohol or drug abuse in the past 2 years, history of cancer, surgery or active illness of the gastro-intestinal tract that might interfere with absorption, intake of any enzyme-affecting drugs in the 30 days prior to the first dosing period, use of any medication, herbal medicine or dietary supplement from 14 days prior to the first dosing (except for occasional paracetamol intake), participation in a trial of an investigational product in the 2 months prior to the first dosing, blood donation in the 2 months prior to the first dosing, history of hypersensitivity or idiosyncrasy to any of the components of the investigational drug, positive drug, alcohol or cotinine test at screening or admission, clinically relevant abnormal laboratory findings, ECG recordings, vital signs or physical or mental findings at screening, and/or major surgery and/or prolonged immobilisation (more than 2 weeks) within the 3 months prior to the screening.

\section{Study drug}

KH176 (or ((S)-6-hydroxy-2,5,7,8-tetramethyl-N-((R)-piperidin-3-yl)chroman-2-carboxamide hydrochloride; Patent WO2014011047 A1) was available as a powder for reconstitution with saline. Placebo was a $\mathrm{NaCl}$ salt/bitrex powder for reconstitution with saline.

\section{Study design}

The trial had a double-blinded, randomized, placebocontrolled, single-centre design. For the SAD part, a partial alternating crossover design and for the MAD part a sequential group design was applied.

In the SAD part the effects of 6 single orally administered ascending doses of $\mathrm{KH} 176$ or placebo were investigated alternately dosed to two groups of 6 healthy male subjects (4 active; 2 placebo per group). Dose escalation 
a

$S A D$ part

\begin{tabular}{|c|c|c|c|c|c|c|c|c|c|}
\hline & \multicolumn{9}{|c|}{ Period } \\
\hline Group & -1 & 1 & 2 & 3 & 4 & 5 & 6 & 7 & 8 \\
\hline I & screening & $\begin{array}{l}\mathrm{D} 1^{*} \mathrm{SD} \\
\text { fasting }\end{array}$ & washout & $\begin{array}{l}D 3^{*} S D \\
\text { fasting }\end{array}$ & washout & $\begin{array}{l}D 5^{*} \mathrm{SD} \\
\text { fasting }\end{array}$ & washout & $\begin{array}{l}\text { D5 SD } \\
+ \text { food }\end{array}$ & FU \\
\hline II & & & $\begin{array}{l}\mathrm{D} 2^{*} \mathrm{SD} \\
\text { fasting }\end{array}$ & washout & $\begin{array}{l}\mathrm{D} 4^{*} \mathrm{SD} \\
\text { fasting }\end{array}$ & washout & $\begin{array}{l}\mathrm{D6} 6^{*} \mathrm{SD} \\
\text { fasting }\end{array}$ & $\mathrm{FU}$ & \\
\hline
\end{tabular}

b

MAD part

\begin{tabular}{|c|c|c|c|c|c|c|c|}
\hline Group & \multicolumn{7}{|c|}{ Period } \\
\hline & -1 & \multicolumn{2}{|c|}{1} & \multicolumn{2}{|c|}{2} & \multicolumn{2}{|c|}{3} \\
\hline III & Screening & $\begin{array}{c}D 1^{*} \\
7 \text { days }\end{array}$ & FU & & & & \\
\hline IV & \multicolumn{3}{|c|}{ Screening } & $\begin{array}{c}\text { D2* } \\
7 \text { days }\end{array}$ & FU & & \\
\hline V & & & \multicolumn{3}{|c|}{ Screening } & $\begin{array}{c}D 3^{*} \\
7 \text { days }\end{array}$ & FU \\
\hline
\end{tabular}

Fig. 1 Study design. a. SAD study. D = Dose; SD = Single dose; FU = follow-up. * Each dose was followed by an interim safety and 24-h PK evaluation, which determined the dose selection for the next dose. A washout period of at least 4 days had to be taken into account. $\mathbf{b}$. MAD study. Gr = group; $D=$ Dose; $F U=$ follow-up * Each dose was followed by an interim 7-days safety and PK evaluation, which determined the dose selection for the next dose

to the next dose level was done after evaluation of the safety and the first 24-h pharmacokinetic results of the previous dose. The single dose part included a food effect investigation; the 100-mg dose was administered following the intake of a high calorie/high fat breakfast to the same subjects as who received this dose in fasting conditions. In the MAD study 3 multiple ascending doses of KH176 were administered for 7 Days to 3 sequential groups of 6 healthy male subjects each (4 active; 2 placebo per group).

\section{Formulation and administration}

KH176 (solution) was administered orally. Single doses of 10, 30, 100, 300, 800 and $2000 \mathrm{mg}$ were administered in the SAD part. Multiple oral doses of 100, 200 and $400 \mathrm{mg}$ were administered b.i.d. for 7 Days in the MAD part. The starting dose for the SAD was 150 fold lower than the no observable adverse effect level (NOAEL) of both dogs and rats. Anticipated exposures at this starting dose were just below the Minimum Anticipated Biological Effect Level (MABEL). Placebo (an in taste and appearance matching oral liquid) was also administered orally on one occasion in the single-ascending dose part and b.i.d. for 7 Days in the MAD part.

\section{Safety assessment}

Safety was assessed using standard vital signs, clinical laboratory results for blood chemistry, haematology and urinalysis, continuous cardiac telemetry and a 12-lead ECG at 1, 2, 4, 6, 8, 12 and $24 \mathrm{~h}$ post dosing. The changes from baseline (pre-dose assessment on Day 1) for body weight, physical examinations, vital signs, ECG-variables and clinical laboratory variables were determined for each time point. Treatment-emergent laboratory and ECG abnormalities as well as adverse events were monitored throughout the study, up to 28 Days after the last study drug intake.

Since preclinical studies in rats demonstrated the occurrence of phospholipidosis, the presence of phospholipidosis was determined by the concentration of didocosahexaenoyl (22:6)-bis(monoacylglycerol) phosphate (di-22:6-BMP) in a midstream urine sample and by electron microscopy of peripheral leucocytes at Day 1 and 7 in the MAD study [14].

\section{Pharmacokinetic analysis}

Plasma samples for pharmacokinetic analyses were taken pre-dose and at $0.5,1,1.5,2,3,6,8,12$, and $24 \mathrm{~h}$ after dosing (SAD) and pre-dose at Day 1, 2, 4, 7, and postdose at Day 1 and Day 7 at 0.5, 1, 1.5, 2, 3, 4, 6, 8, $12 \mathrm{~h}$ after dosing (MAD). All samples were stored at $<-70{ }^{\circ} \mathrm{C}$ until analysis. Quantification of $\mathrm{KH} 176$ and its metabolite $\mathrm{KH} 176 \mathrm{~m}$ were performed using a validated LC-MS/ MS method with good ( $\max 15-20 \% \mathrm{CV}$ ) selectivity, precision and accuracy, little carry over effect, good stability of both the solutions and the samples.

For mean value calculations, all values below the limit of quantification (LOQ) were set to zero. If $<50 \%$ of the values at a given time point were below the LOQ (BLQ), these values were set to zero for calculation of the mean value. If $>50 \%$ of the values at a given time point were BLQ, no mean value was calculated. The noncompartmental pharmacokinetic analysis was performed using Phoenix, Version 6.3 (Pharsight Corporation, Mountain View, CA, USA). Plasma concentration-time profiles of $\mathrm{KH} 176$ and its metabolite $\mathrm{KH} 176 \mathrm{~m}$ were determined for the SAD and MAD part and for trough concentrations in the MAD part. The PK parameters 
were calculated on the basis of the actual blood sampling time points relative to dosing. Selected pharmacokinetic parameters $\left(C_{\max }, t_{\max }, t_{1 / 2}, A U C_{\text {last }}\right.$ and $\mathrm{AUC}_{0 \text {-inf }}$ ) following single-dose administration and selected pharmacokinetic parameters $C_{\max }, t_{\max }, t_{1 / 2}$, $\mathrm{AUC}_{\text {tau }}$, the accumulation factor $\left(\mathrm{R}_{\mathrm{acc}}\right)$ and time to reach steady state) following multiple dose administration were determined. In urine, the percentage of dose excreted in urine was determined for the singleand multiple-dose administration.

\section{Pharmacodynamic analysis}

Blood samples for pharmacodynamic analyses were taken pre-dose and at 3, 6 and 24 h post dosing at Day 7 (MAD). All samples were stored in $-80{ }^{\circ} \mathrm{C}$ until analysis. Quantification of oxidized and reduced glutathione was performed at York Bioanalytical solutions using a previously described validated method [15]. Change from baseline in the concentrations of oxidized and reduced glutathione (GSH/GSSG) was calculated.

\section{ECG post-hoc analysis}

During the interim evaluations at dose escalation, machine-read ECGs and telemetry in the clinic indicated a prolongation of the corrected QT interval by KH176, as indicated by QTcB, the Bazett corrected QT interval, particularly strong at high dosages of the compound. Correction of the QT interval is indicated to allow comparison between QT values over different heart rates, since the QT interval shortens with higher heart rates. In the post-hoc analysis, we have used the Fridericia formula $\left(\mathrm{QTcF}=\mathrm{QT} / \mathrm{RR}^{1 / 3}\right)$, which is more reliable compared to the QTcB outside the range of 60$100 \mathrm{bpm}$. The goal of this post-hoc optimization study was to repeat the machine-read ECG assessment with a highly automated computer-assisted approach where in addition to the re-evaluation of standard intervals (PR, QRS and QT intervals), a set of parameters describing repolarization morphology were considered. The morphology indices were the TpTe interval (interval from the $\mathrm{T}$ wave apex to the end of the $\mathrm{T}$ wave), the $\mathrm{TpTe} / \mathrm{QT}$ index (the ratio between the TpTe interval and the QT interval), Tamp (the amplitude in microvolt units of the $\mathrm{T}$ wave) and TSym (an index of repolarization morphology based on the symmetry of the $\mathrm{T}$ wave).

ECGs were digitally recorded using a Schiller AT104 ECG machine $(500 \mathrm{~Hz}, 1 \mu \mathrm{V})$. Cardiac interval measurements were performed on the Global Superimposed Median Beat (GSMB), a methodology that allows measurements that consider each of the 12 individual median beats [16]. This measurement methodology ensures that the PR, QRS, and QT interval are measured from the earliest onset in any lead to the latest deflection in any lead. Cardiologist overview of the computer-based measurements is based on the superimposed (overlapped) display of the individual median beats, to assure measurements are performed at the earliest onset of any viable lead to the latest offset of any viable lead.

The RR interval used for heart-rate correction of the QT interval (QTcB) was based on all the beats in the ten second recording.

Other cardiac parameters were computed from the 12lead vector magnitude VM (e.g. the square root of the sum of squares at each digital sample) computed from the individual median beats. On the VM lead, the apex of the $\mathrm{T}$ wave is placed and used to compute the TpTe interval (using the end of the $\mathrm{T}$ wave from the GSMB) and the $\mathrm{T}$ wave amplitude (height in microvolt of the $\mathrm{VM} \mathrm{T}$ peak from the isoelectric line). Additional file 1: Figure S1A shows an example with all the calipers involved: the GSMB leads are drawn in black and the VM lead is drawn in green. $\mathrm{T}$ wave symmetry index was computed using a proprietary approach based on Gaussian Mesa function modeling (GMF) of the repolarization waves [17]. Briefly, the ascending and descending phases of the VM T wave are modeled by two independent half-Gaussian curves (see Additional file 1: Figure S1). The standard deviations of these functions ( $\sigma 1$ and $\sigma 2)$ and indicators of the ascending/descending speed and their ratio is an index of symmetry (TSym $=\sigma 1 / \sigma 2$; $\mathrm{TSym}=1$ for a perfectly symmetric $\mathrm{T}$ wave, $\mathrm{TSym}<1$ for slow-ascending/fast-descending $\mathrm{T}$ waves, $T>1$ for fastascending/slow-decending $\mathrm{T}$ waves).

For the pre-dose (baseline) ECGs, all parameters per time point were calculated as the averages of the triplicate ECGs. Computation of QTcB and QTcF was performed using the RR interval averaged from the total ECG acquisition duration (10 s) and in the case of the triplicate ECGs is based on the average QT and average HR of the replicate ECGs.

The over-reading cardiologist provided a clinical interpretation for each ECG at each time point. Each ECG was classified as Normal, Abnormal Clinically Insignificant (ACI), or Abnormal Clinically Significant (ACS), based on absolute changes in QTc interval (> $450 \mathrm{~ms})$, changes from baseline $(>60 \mathrm{~ms})$ and the presence of secondary arrhythmias. Results of this post-hoc analysis were used for an exposure-response evaluation for the changes in the electrophysiological parameters as a function of the concentration of KH176.

\section{Ethics}

All studies were conducted at the Drug Research Unit Ghent in accordance with the Declaration of Helsinki and the Good Clinical Practice guidelines established by the International Conference on Harmonization (ICH). An independent medical ethical committee approved the 
protocol (University Hospital of Ghent). All participants have signed informed consent prior to their enrolment.

This trial has been registered at clinicaltrials.gov prior to the conduction of the study (NCT02544217) and retrospectively at 23-06-2017 (ISRCTN43372293).

\section{Statistical analysis}

The tolerability and safety data were compared between KH176 and placebo using descriptive summary statistics. Pharmacokinetic and pharmacodynamic parameters were analyzed by treatment group on the per-protocol set and summarized using descriptive statistics. Dose proportionality of $\log$ transformed $\mathrm{C}_{\max }$ and AUC values was explored graphically. The effect of food on the pharmacokinetics was explored by calculating the geometric means and $90 \%$ confidence interval of the ratio (fed/fasted) for AUC and $\mathrm{C}_{\max }$. The pharmacodynamic endpoints were analyzed descriptively by treatment group on the per-protocol set as a change from baseline. Pharmacokinetic-effect modelling of PK/ECG data was explored visually and by an exposure-response evaluation for the changes in the electrophysiological parameters as a function of the concentration of KH176.

\section{Results}

\section{Study population}

For the SAD part of the study, 14 healthy male subjects divided in two groups of 7 subjects were included. Two subjects prematurely withdrew from the study after dosing period 2 for non-medical reasons. For the MAD part of the study, 18 healthy male subjects divided in 3 groups of 6 subjects were included. Two subjects prematurely withdrew from the study for non-medical reasons and were replaced. There were no protocol violations in either part of the study that excluded subjects from the analysis set(s) and, therefore, all subjects included in this study were evaluable for pharmacokinetics, pharmacodynamics, safety, and tolerability. For demographics, see Table 1.

\section{Safety and tolerability}

Following administration of a single dose of KH176 to subjects in the fasted state (SAD study), a total of 43 adverse events (AEs) were reported by 14 subjects (56\%; Additional file 2: Table S1A). The majority of AEs (35) was rated as mild in severity whereas there were $6 \mathrm{mod}-$ erate and 2 severe AEs. Up to and including the $800 \mathrm{mg}$ dose, no relationship between the presence of placebo or KH176, the dose of KH176 and the number or the severity of reported AEs could be discerned (Additional file 2: Table S1). However, 28 of the 43 reported AEs were reported by subjects in the highest dose group of which 6 and 2 were of moderate and severe severity, respectively. The 2 severe AEs were nausea and headache. Following administration of placebo, a total of 7 AEs were reported by 3 subjects (25\%). Of note, 4 of these AEs were rated as moderate in severity. Headache was the most frequently reported $\mathrm{AE}$ with a total of 7 subjects including 2 placebo subjects. The majority of AEs occurred incidentally, i.e., was reported by only 1 or 2 subjects. Adverse events reported by more than 2 subjects and not by placebo subjects were psychiatric symptoms, dizziness, oral paraesthesia and prolonged QT at the electrocardiogram and telemetry. All these events occurred in the highest dose group (2000 $\mathrm{mg}$ dose) and were reported by 3 subjects each.

Following administration of a multiple doses of KH176 (MAD study), a total of 29 AEs were reported by 10 subjects (83.3\%; Additional file 2: Table S1B). The majority of AEs (27) was rated as mild in severity whereas there were 2 moderate AEs, one each in the 200- and 400-mg dose groups. No relationship to dose and the number of reported AEs or severity could be discerned. Headache was the most frequently reported $\mathrm{AE}$ with a total of 11 subjects including 5 placebo subjects reporting this AE. Except for skin irritation, all AEs occurred incidentally, i.e., were reported by only 1 or 2 subjects in the KH176 treated subjects. Skin irritation was also reported by 2 placebo subjects and, therefore, no $\mathrm{AE}$ was reported by more than 2 KH176-treated subjects and not by placebo subjects.

The level of di-22:6-BMP was variable at baseline. There was no increase in di-22:6-BMP for the treatment groups versus placebo (change from Day 1 to Day 7 $0.74-2.50 ;-1.44-1.32$ and $-0.69-1.46 \mathrm{ng} / \mathrm{mg}$ creatinine for group III, IV and V respectively and -0.92-4.31 ng/ $\mathrm{mg}$ creatinine for placebo). No increased prevalence of the presence of phospholipidosis in either granulocytes or monocytes as evaluated by electron microscopy was present in the $\mathrm{KH} 176$-treated group versus the placebotreated group.

\section{Pharmacokinetic analysis SAD study}

The plasma concentrations-time profiles of KH176 (Fig. 2a) showed that the median $t_{\max }$ was between 0.75 and $1.5 \mathrm{~h}$ after administration and varied little with dose. Thereafter, the KH176 concentrations decreased in a biphasic way. The $t_{1 / 2}$ was approximately $10 \mathrm{~h}$ and varied little among dose groups (Table 2). The elimination phase following administration of a dose of $10 \mathrm{mg}$ was not well characterized and, therefore, $t_{1 / 2}$ could not be estimated for this dose only.

The shape of the plasma concentration-time profiles of KH176m (Fig. 2b) resembled that of the parent compound but concentrations were lower. $C_{\max }$ was reached at the same time or slightly later when compared to KH176 (Additional file 2: Table S2). Thereafter, KH176m 
Table 1 Summary of demographic characteristics for subjects included in the SAD and MAD study

\begin{tabular}{|c|c|c|c|c|c|c|}
\hline & \multicolumn{2}{|l|}{ SAD } & \multicolumn{4}{|l|}{ MAD } \\
\hline & Group I $(N=7)^{a}$ & Group $\|(N=7)^{a}$ & $\begin{array}{l}\text { Group III } 100 \mathrm{mg} \\
\text { BID }(N=4)\end{array}$ & $\begin{array}{l}\text { Group IV } 200 \mathrm{mg} \\
\operatorname{BID}(N=4)\end{array}$ & $\begin{array}{l}\text { Group } \vee 400 \mathrm{mg} \\
\operatorname{BID}(N=4)\end{array}$ & $\begin{array}{l}\text { Placebo } \\
(N=6)\end{array}$ \\
\hline \multicolumn{7}{|l|}{ Age (years) } \\
\hline Mean (SD) & $30.4(10.5)$ & $32.0(11.3)$ & $40.8(13.0)$ & $34.5(11.6)$ & $44.0(8.2)$ & $44.7(9.8)$ \\
\hline Median & 28.0 & 31.0 & 44.0 & 35.0 & 43.0 & 48.5 \\
\hline Range & $(22-52)$ & $(18-45)$ & $(24-51)$ & $(23-45)$ & $(37-53)$ & $(28-54)$ \\
\hline \multicolumn{7}{|c|}{ Height $(\mathrm{cm})$ at screening } \\
\hline Mean (SD) & $178.07(6.56)$ & $176.01(8.11)$ & $184.63(2.81)$ & $181.75(5.25)$ & $175.70(3.18)$ & $175.55(8.25)$ \\
\hline Median & 177.50 & 177.00 & 184.90 & 183.75 & 176.70 & 171.60 \\
\hline Range & $(165.7-184.5)$ & $(163.0-187.5)$ & $(181.5-187.2)$ & $(174.0-185.5)$ & $(171.1-178.3)$ & $(170.4-191.5)$ \\
\hline \multicolumn{7}{|c|}{ Weight $(\mathrm{kg})$ at screening } \\
\hline Mean (SD) & $82.86(10.56)$ & $74.29(12.61)$ & $89.05(11.84)$ & $77.85(11.96)$ & $81.10(4.82)$ & $72.03(6.06)$ \\
\hline Median & 80.00 & 78.60 & 89.30 & 80.30 & 82.80 & 72.30 \\
\hline Range & $(69.8-100.0)$ & $(55.2-88.6)$ & $(77.2-100.4)$ & $(61.2-89.6)$ & $(74.2-84.6)$ & $(62.6-78.8)$ \\
\hline \multicolumn{7}{|c|}{$\mathrm{BMI}\left(\mathrm{kg} / \mathrm{m}^{2}\right)$ at screening } \\
\hline Mean (SD) & $26.09(2.51)$ & $24.01(4.01)$ & $26.10(2.74)$ & $23.60(3.82)$ & $26.28(1.67)$ & $23.43(1.98)$ \\
\hline Median & 26.10 & 22.80 & 26.25 & 24.90 & 26.70 & 23.50 \\
\hline Range & $(22.5-29.4)$ & (19.0-29.6) & $(23.1-28.8)$ & $(18.2-26.4)$ & $(23.9-27.8)$ & $(21.2-26.6)$ \\
\hline \multicolumn{7}{|l|}{ Race } \\
\hline White & 7 (100.0\%) & 7 (100.0\%) & $4(100.0 \%)$ & $4(100.0 \%)$ & $4(100.0 \%)$ & $6(100.0 \%)$ \\
\hline
\end{tabular}

plasma concentrations decreased in a biphasic way and the elimination was characterized by a $t_{1 / 2}$ of approximately $16 \mathrm{~h}$ without any notable effect of dose.

In the presence of food, the absorption of KH176 was slower as indicated by a median $t_{\max }$ that shifted from approximately $1 \mathrm{~h}$ in fasted condition to $2.5 \mathrm{~h}$ in fed condition for both analytes (Table 2). Exposure under fed conditions in terms of $\mathrm{AUC}_{0 \text {-inf }}$ increased slightly for KH176 whereas that to KH176m decreased slightly $\left(\mathrm{AUC}_{0 \text {-inf }} 1.28\right.$ (90\%CI 1.12-1.45) for $\mathrm{KH} 176$ and 0.90 (90\%CI $0.57-1.41)$ for $\mathrm{KH} 176 \mathrm{~m})$. The $\mathrm{t}_{1 / 2}$ was not affected by food.

A graphical exploration for dose-proportionality of the pharmacokinetics of KH176 indicated that with increasing single dose there was a more than proportional in-

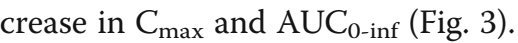

Regardless of the dose and when combining KH176 and $\mathrm{KH} 176 \mathrm{~m}$, approximately $16 \%$ of the administered dose was excreted in urine. Unchanged KH176 accounted for approximately $12 \%$.

\section{MAD study}

Visual inspection of the mean trough concentrationtime curves indicated that steady concentrations of KH176 were reached by Day 4 of dosing (of note: Day 4 was the first time point of measurement of trough values; Fig. 2c).
The plasma concentration-time profiles of KH176 after single- and multiple-dose administration were similar. Peak concentrations were attained between 1 and $2 \mathrm{~h}$ after drug administration (Table 2). Following attainment of $\mathrm{C}_{\max }$, the $\mathrm{KH} 176$ plasma concentrations declined rapidly. KH176 accumulated as shown by values for the accumulation index between doses varying from 2.17 to 2.65 .

After multiple dosing, the shape of the plasma concentration-time profiles of $\mathrm{KH} 176 \mathrm{~m}$ resembled that of parent compound but concentrations were lower (Fig. 2d). On Day 7, $\mathrm{C}_{\max }$ was reached at approximately the same time when compared to KH176. After multiple dosing, the accumulation of the metabolite $\mathrm{KH} 176 \mathrm{~m}$ was less pronounced when compared to that of KH176 as indicated by values for the accumulation index varying from 1.19 to 1.86 .

A graphical exploration for dose-proportionality of the pharmacokinetics of KH176 after multiple dosing indicated that with increasing multiple doses there was a more than proportional increase in $C_{\max }$ and $\mathrm{AUC}_{\mathrm{tau}}$, which was most pronounced at the $400 \mathrm{mg}$ dose (Fig. 3).

On Day 7 and when combining KH176 and $\mathrm{KH} 176 \mathrm{~m}$, the \%dose excreted in urine varied from 18.0 to $25.1 \%$ between doses. Unchanged KH176 accounted for 14.0 to $18.1 \%$. 

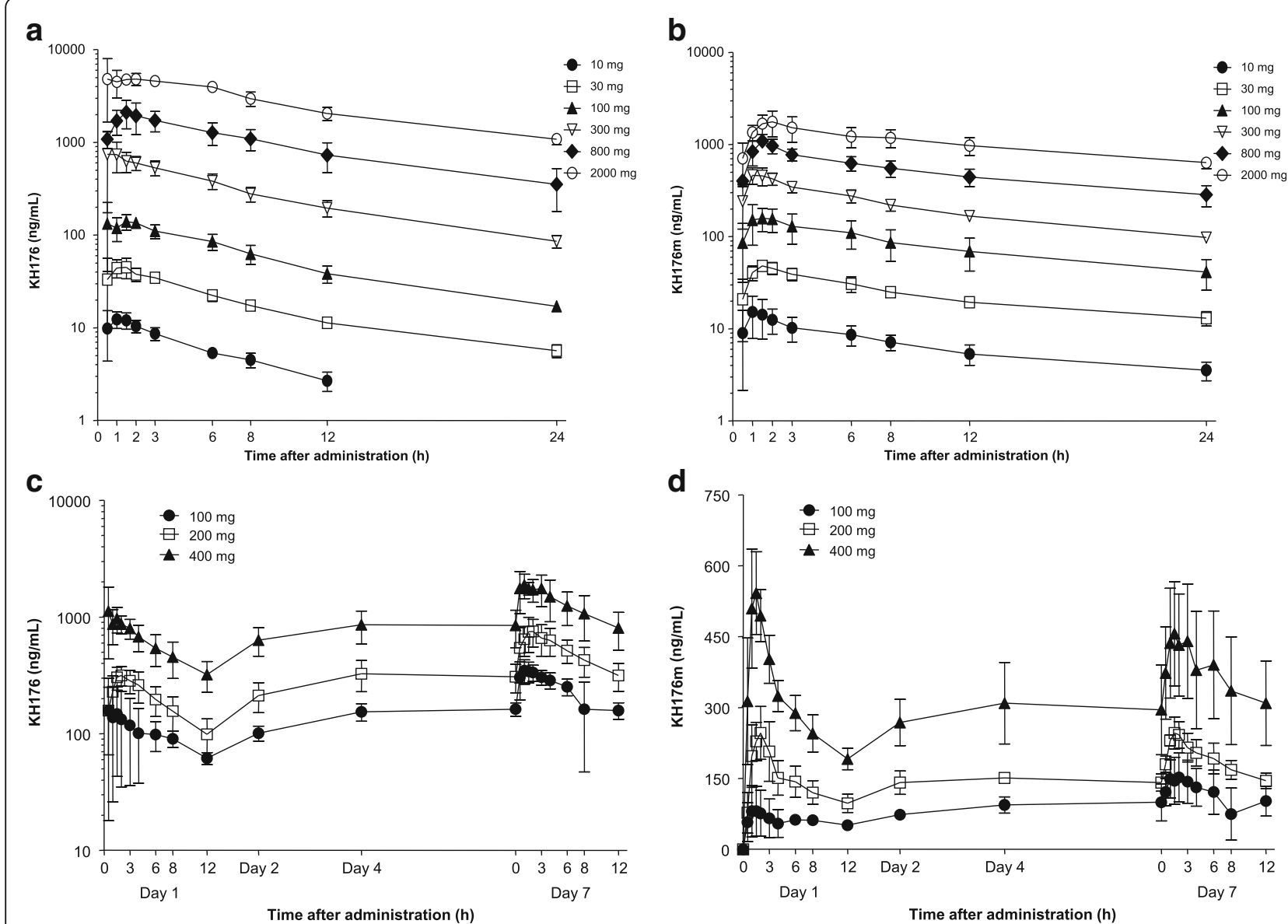

Fig. 2 Mean plasma concentrations of $\mathrm{KH} 176$ and its metabolite $\mathrm{KH} 176 \mathrm{~m}$ after single and multiple dose administration to healthy subjects. a. Plasma-concentration/time curve of KH176 for the SAD study (fasted state). b. Plasma-concentration/time curve of KH176m for the SAD study (fasted state). c. Plasma-concentration/time curve of $\mathrm{KH} 176$ for the MAD study. d. Plasma-concentration/time curve of KH176m for the MAD study. Loglinear scale

\section{Pharmacodynamic analysis}

No significant alterations in the GSH/GSSG ratio were observed.

\section{Posthoc analysis QTc prolongation}

A QTcF prolongation was present after single-dose administration of 800 and $2000 \mathrm{mg}$ KH176 (see Additional file 3: Figure S3 for a representative example). The largest median baseline increase observed was $46.8 \mathrm{~ms}$ ( $1 \mathrm{~h}$ post-dose), whereas the largest individual change was $64.7 \mathrm{~ms}$ (2000 mg group; Additional file 4: Figure S2). This QTcF prolongation was associated with moderate but clear changes of morphology, namely a reduction of the $\mathrm{T}$ wave amplitude, a prolongation of the TpTe interval (in both absolute terms and relatively to the QT interval), and the symmetry (shape) of the $\mathrm{T}$ wave. At lower doses, KH176 does not seem to affect repolarization. Changes were also observed on other cardiac intervals: the QRS interval increased but only at a dose of $2000 \mathrm{mg}$, whereas the PR interval increased progressively also at lower doses of KH176.
The MAD part of the study showed the same effects, particularly for the $400 \mathrm{mg}$ dose group (Additional file 4: Figure S2). A dose of $100 \mathrm{mg}$ b.i.d. had no effect on QTcF and the time curve for this dose was indistinguishable from placebo. On Day 4 and later, administration of $200 \mathrm{mg}$ b.i.d. increased QTcF and a median maximum change from baseline was observed on Day 5 of $13 \mathrm{msec}$. A further increase in QTcF was observed with a dose of $400 \mathrm{mg}$ b.i.d. and the median maximum change from baseline at trough of $29 \mathrm{msec}$ was observed on Day 6 .

Clinically relevant changes were observed in at the peak concentrations in 2 individuals in the $2000 \mathrm{mg}$ subgroup and did not include appearance of notched and/or bumps on any of the T-waves (all leads).

When the change in the TpTe interval is correlated to the KH176-exposure, a clear dose-dependency is observed (Fig. 4). In exposures lower than $500 \mathrm{ng} / \mathrm{ml}$, no significant prolongation of the TpTe interval compared to placebo was observed. When increasing the dose and the plasma concentration, the TpTe interval increases. When the highest (non-tolerated) dose was 
Table 2 Summary of plasma pharmacokinetic variables of KH176

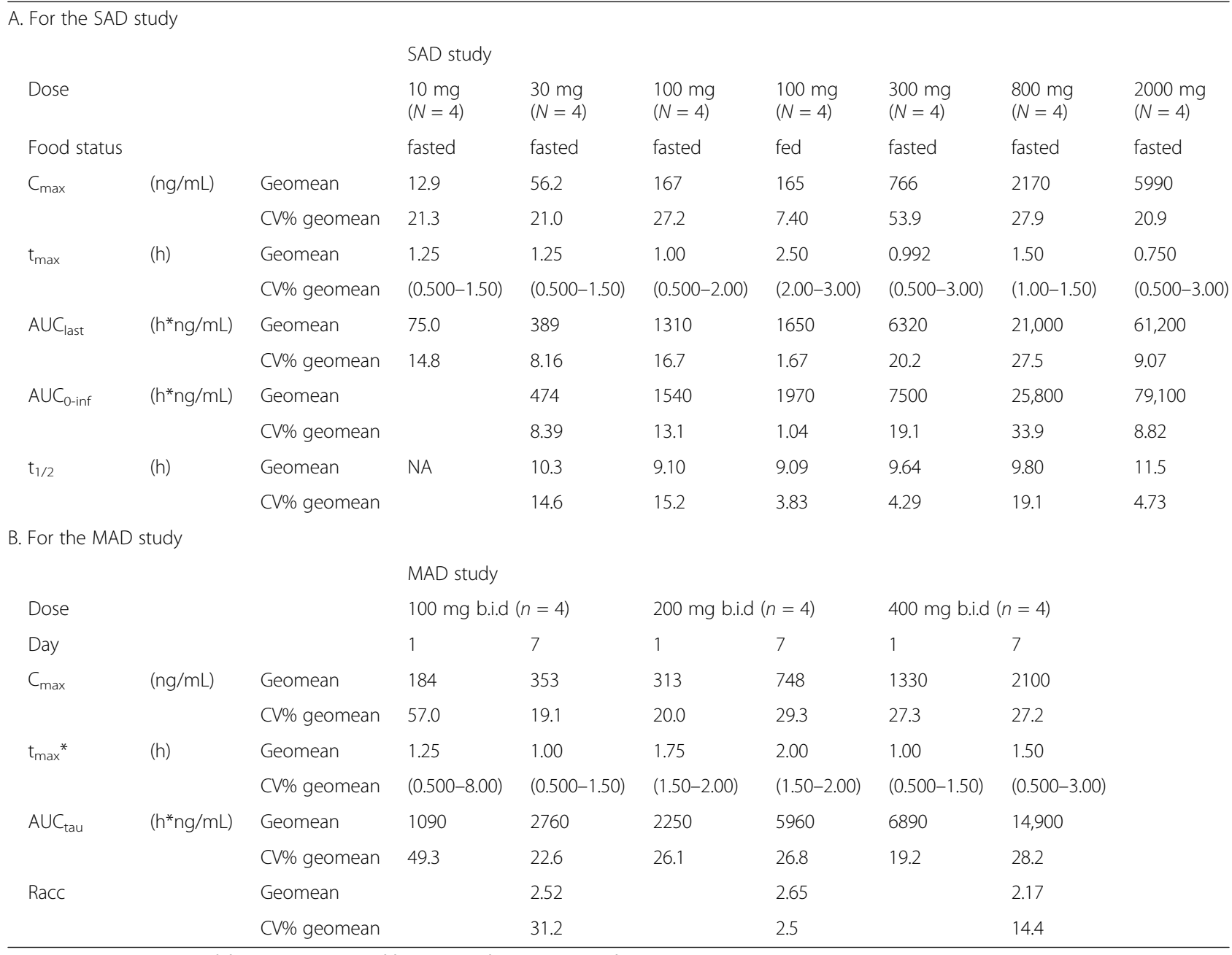

Geomean geometric mean, $h$ hour, NA not assessable, $R$ accumulation ratio, *median (range)

administered (2000 mg), all subjects have a prolonged TpTe interval.

\section{Discussion}

Mitochondrial disorders are a devastating group of disorders for which there is an urgent need for treatment development. In preclinical studies, KH176 showed promising properties in ameliorating the viability and phenotype of cells and mice affected by mitochondrial disease. The objective of this study was to evaluate the tolerability, safety, pharmacokinetics and pharmacodynamics of single- and multiple-ascending doses of KH176 in healthy male subjects.

KH176 was well tolerated in doses up to $800 \mathrm{mg}$ SD and $400 \mathrm{mg}$ b.i.d.. Headache was the most frequently reported AE in both the $\mathrm{KH} 176$ - and the placebo treated groups. Although there was no clear dose-response relationship for any of the adverse effects after single and multiple dose administration, unforeseen markedly more severe adverse events were reported after a single dose of $2000 \mathrm{mg}$. At this dose, which is a factor 10 above the anticipated daily human efficacious dose of $200 \mathrm{mg} /$ day, nausea, vomiting, dizziness and psychiatric disturbances were reported, along with a prolonged corrected QT time.

The shape of the plasma concentration-time profiles of KH176 and its metabolite KH176m were similar after single- and multiple-dose administration. The pharmacokinetics of KH176 showed several aspects. In particular, the pharmacodynamics of KH176 were characterized by a i) median $t_{\max }$ between 0.75 and $2.0 \mathrm{~h}$, ii) terminal $t_{1 / 2}$ of about $10 \mathrm{~h}$, iii) biphasic elimination, and a iv) more than dose proportional increases in both $\mathrm{C}_{\max }$ and AUC.

In vitro, KH176 is metabolized by CYP3A4 and excreted by the PgP efflux pump. We did not observe signs for auto-induction in this study such as a decline in predose concentrations. Without wishing to be bound by any theory, the more than proportional increase with dose could be caused by saturation of the PgP efflux pump for which KH176 is a substrate, as evidenced by 

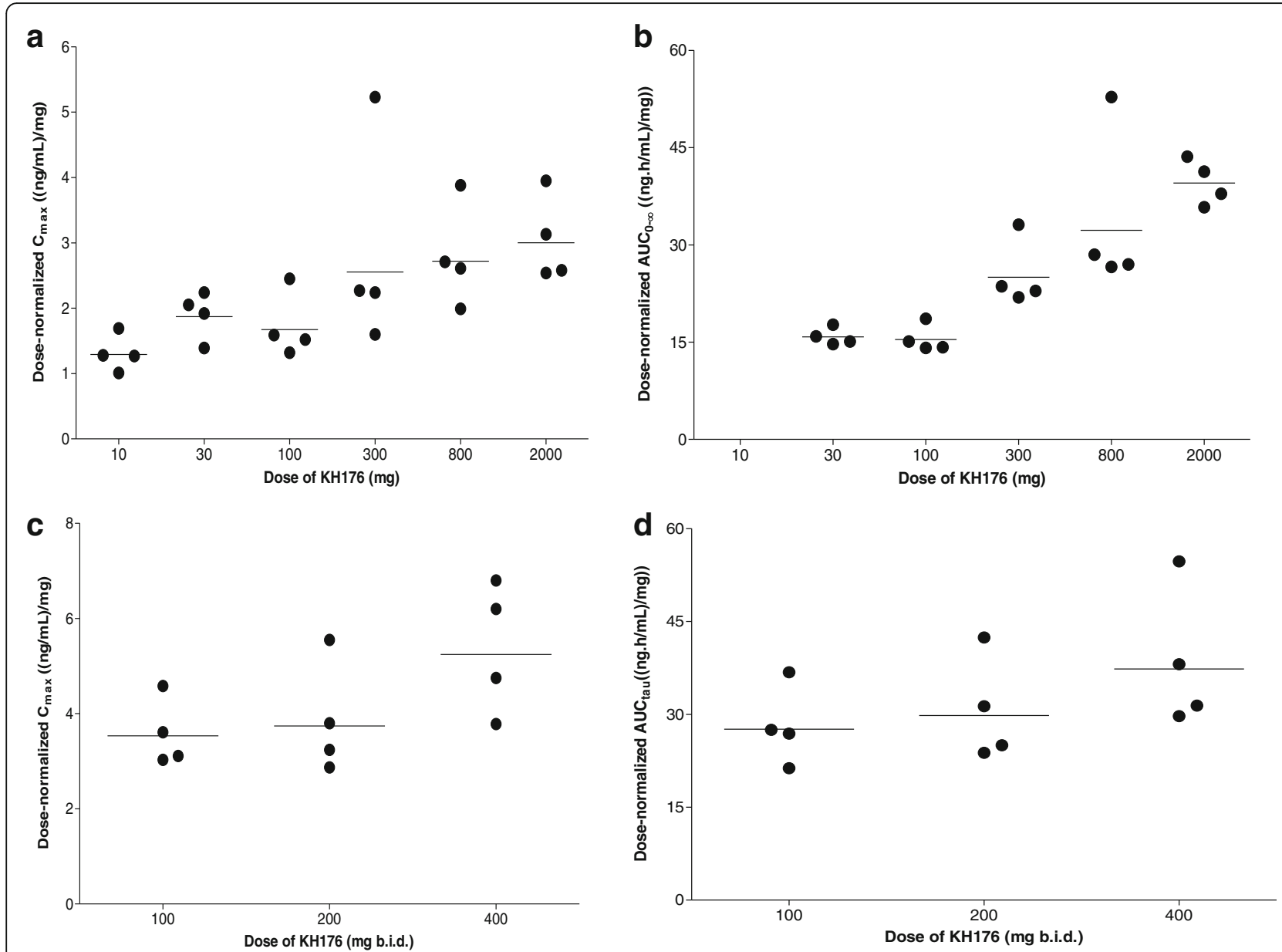

Fig. 3 Dose-normalized individual values for $C_{\max }$ and $A \cup C_{0 \text {-inf }}$ of $K H 176$ for the SAD and the MAD study. a. Dose normalized $C_{\max }$ for the SAD study. b. Dose normalized $A \cup C_{0 \text {-inf }}$ for the SAD study. c. Dose normalized $C_{\max }$ for the MAD study. $\mathbf{d}$. Dose normalized $A \cup C_{0 \text {-inf }}$ for the MAD study. The horizontal lines depict the geometric mean value

the fact that half-life and clearance doesn't change with dose, but rather the availability seems to change with dose. Up to $25.1 \%$ of an administered dose is excreted via urine after multiple dosing. In steady state conditions at $100 \mathrm{mg}$ BID dosing the maximum concentration reached ranges within 303-458 ng/mL for KH176 and 89.4-204 ng/mL for $\mathrm{KH} 167 \mathrm{~m}$ (hence the maximum concentrations of these active moieties ranged within 392.4-662 ng/mL).

Based on the results of the multiple-dose administration, steady state was reached at the first time point of measurement of trough concentrations (Day 4). Based on the estimated $t_{1 / 2}$, it is expected that steady state will be reached after 2 to 3 Days of dosing. KH176 was well tolerated in the presence of food, and the tolerability and safety profile in the presence and absence of food was similar. Although the point estimates (as well as the sample covariance) for both $\mathrm{C}_{\max }$ and $\mathrm{AUC}_{0 \text {-inf }}$ did not completely remain within the usually accepted range of 0.8 to 1.25 (Table 2), no special measures are warranted regarding the intake of KH176.
KH176 clearly modifies cardiac repolarisation in a dose-dependent manner. QTcF prolongation was present after single-dose administration of 800 and $2000 \mathrm{mg}$ and after multiple doses of $400 \mathrm{mg}$ of KH176 b.i.d.. Post-hoc studies showed that this QTcF prolongation was associated with changes in morphology and other cardiac intervals. Detailed analysis of the ECGs during the single-dose administration of 200, 100, 30 and $10 \mathrm{mg}$ and multiple oral doses of 100 and $200 \mathrm{mg}$ b.i.d. showed no cardiac electrophysiological abnormalities. Moreover, the changes in the QTcF interval in the $800 \mathrm{mg}$ KH176 single dose and the $400 \mathrm{mg}$ b.i.d KH176 dose did not reach the threshold for clinical relevance. The KH176 related changes in cardiac repolarisation include a reduction of $\mathrm{T}$ wave amplitude, a prolongation of the $\mathrm{TpTe}$ interval and a reduction of the $\mathrm{T}$ wave symmetry index. The reduction in $\mathrm{T}$ wave amplitude is largely explained by changes in heart rate and also seen in the placebo group. The prolongation of the TpTe interval and the reduction on the symmetry index indicate a prolongation of the 


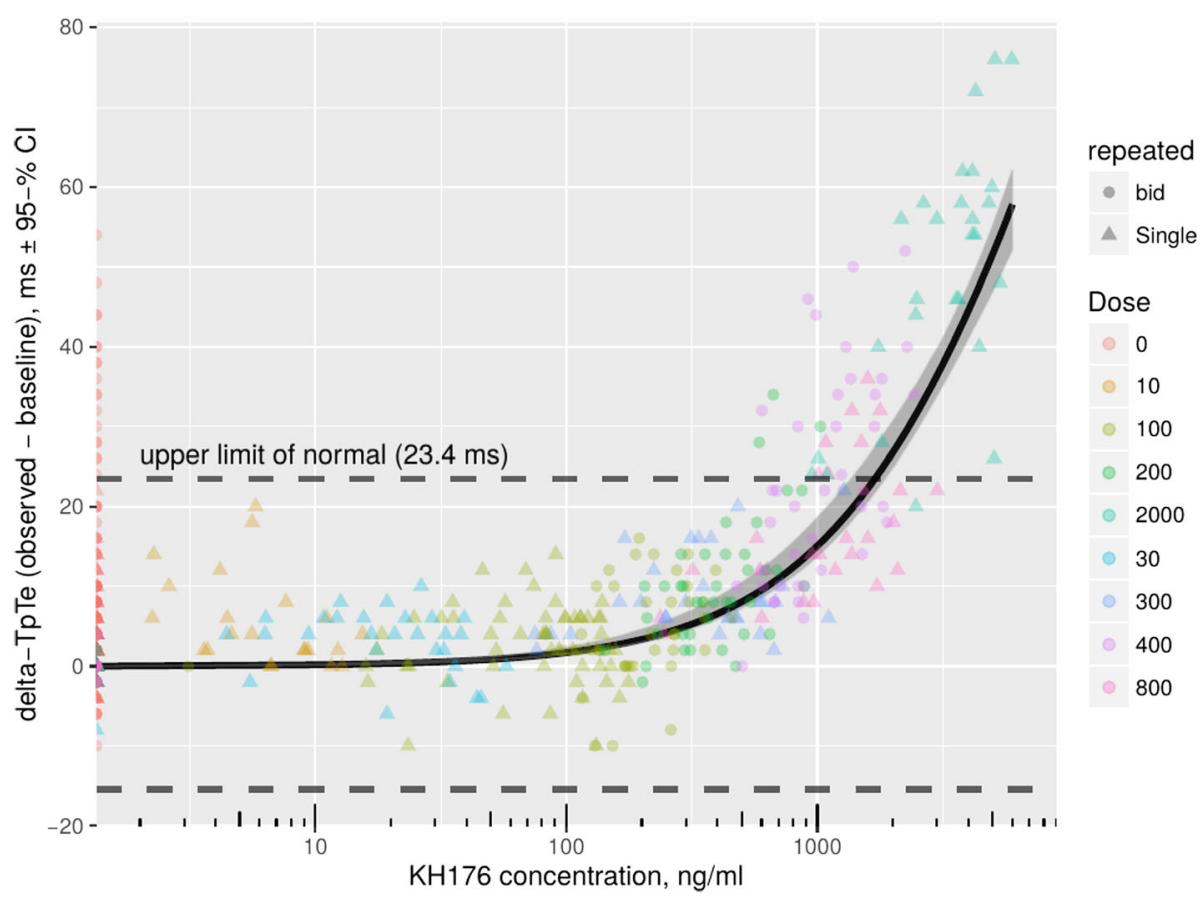

Fig. 4 Exposure-response analysis of KH176 plasma concentrations and a change from baseline for ECG derived TpTe intervals. The upper limit of normal $(23.4 \mathrm{~ms}$ ) is derived from the $95 \%$ confidence interval of the pre-dose values. In healthy subjects a dose of 100 mg BID resulted in maximum concentrations ranging from 303 to $458 \mathrm{ng} / \mathrm{mL}$

descending phase of the $\mathrm{T}$ wave. With pure hERG blockade, there is a direct relationship between increasing plasma drug concentrations and the risk for torsades. Importantly, although the specific $\mathrm{T}$ wave morphology changes observed in this study have been associated with the hERG potassium channel blockade [18], the $\mathrm{IC}_{50}$ of hERG blocking for KH176 was 3 times higher than the $\mathrm{C}_{\max }$ for the highest dose.

The clinical relevance of these changes in cardiac electrophysiology are not clear yet. Notably, there were 2 subjects with a potentially clinically relevant change $(>60 \mathrm{~ms})$ at the peak concentration after administration of $2000 \mathrm{mg}$ but no signs of arrhythmias or severe morphologic changes (such as $\mathrm{T}$ wave bumps, notches, etc) were observed. One should be cautious when translating these healthy volunteer results to a patient population. Since the sensitivity to torsades de pointes also depends on many other factors including cardiovascular disease, alcoholic liver disease, obesity, hypertension, and/or electrolyte disturbances $[19,20]$, high concentrations (e.g. more than a total daily dose of $1000 \mathrm{mg}$ ) of KH176 preferably should not be given to patients with any of these risk factors. Moreover, until dose adjustments for patients with concomitant medication (also metabolized by CYP3A4) are determined, high concentrations (e.g. more than a total daily dose of $1000 \mathrm{mg}$ ) of KH176 should preferably not be given to these patients either since unpredictable pharmacokinetics will possibly lead to plasma concentrations of KH176 above the currently defined safety threshold.

Since the mechanism of KH176 is based on correction of an abnormal redox balance, as expected, we did not observe any pharmacodynamic changes in the healthy male volunteers.

Comparing human exposure to mouse exposure in the in vivo mouse studies and benchmarking human exposure to in vitro activity of $\mathrm{KH} 176$ and metabolites indicates that $100 \mathrm{mg}$ b.i.d. dosing results in a putatively efficacious exposure to be tested in the following Proof of Concept study.

\section{Conclusion}

We conclude that administration of single doses up to and including $800 \mathrm{mg}$ or multiple doses up to $400 \mathrm{mg}$ b.i.d. for 7 days of KH176, a new small redox-modulating molecule developed to treat mitochondrial(-related) diseases and conditions, is safe and well tolerated in healthy male volunteers. Although doses above the anticipated human efficacious dose could lead to prolongation of the QTc interval with T-wave abnormalities, these changes did not reach the threshold for clinical relevance in these men. We have recently performed a phase 2 study in m.3243A > G carriers, administering the anticipated efficacious dose which did not lead to changes in cardiac electrophysiology in healthy men (100 mg KH176 b.i.d). Based on the study reported here, patients with cardiac abnormalities were excluded for safety reasons. 


\section{Additional files}

Additional file 1: Figure S1. Posthoc ECG assessment methodology. A. Example of an ECG adapted on the Global Superimposed Median Beat (GSMB). B. T wave symmetry index was computed by modeling the $T$ wave in two independent half-Gaussian curves. The standard deviations of these functions ( $\sigma 1$ and $\sigma 2$ ) and indicators of the ascending/descending speed. (PDF 143 kb)

Additional file 2: Table S1. Summary of treatment-emergent adverse events by system organ class and preferred term. Table S2. Summary of plasma pharmacokinetic variables of KH176m. Table S3. Largest median an largest individual increase in the ECG parameters for the SAD and the MAD study. (DOCX $36 \mathrm{~kb}$ )

Additional file 3: Figure S3. Representative example of the changes in the intervals in an individual in the $2000 \mathrm{mg}$ group. (PNG $63 \mathrm{~kb}$ )

Additional file 4: Figure S2. Posthoc ECG assessment results. A. Change in QTCF (median increase from baseline; SAD study). B. Change in TpTe (median increase from baseline; SAD study). C. Change in the T-wave symmetry index (median increase from baseline; SAD study). D. Change in QTCF (median increase from baseline; SAD study). E. Change in TpTe (median increase from baseline; MAD study). F. Change in the T-wave symmetry index (median increase from baseline; MAD study). (PDF $341 \mathrm{~kb}$ )

\section{Abbreviations}

ACl: Abnormal clinically insignificant; ACS: Abnormal clinically significant; AE: Adverse events; b.i.d.: bis in die (two times daily); BLQ: Below limit of quantification; GSMB: Global superimposed median beat; HR: Heart rate; LOQ: Limit of quantification; MABEL: Minimum anticipated biological effect level; MAD: Multiple ascending dose; MD: Multiple dose; NOAEL: No observed adverse effects level; QTcB: Bazett's corrected QT interval; QTcF: Fridericia's corrected QT interval; SAD: Single ascending dose; SD: Single dose; Tamp: the amplitude in microvolt units of the T wave; TEAE: Treatment-emergent adverse events; TpTe interval: interval from the T wave apex to the end of the T wave; TpTe/QT index: the ratio between the TpTe interval and the QT interval; TSym: index of repolarization morphology based on the symmetry of the T wave

\section{Acknowledgements}

The authors are grateful to the excellent support of the Clinical Trial Unit Gent, the Khondrion team and its advisors.

\section{Funding}

The work of SK was supported by the Zeldzame Ziekten Fonds (ZZF). JS is supported by grants from ZonMW PMRare (113302003), Energy4All, Join4Energy, Ride4Kids and the Tim Foundation.

\section{Availability of data and materials}

Available from the corresponding author on reasonable request.

\section{Authors' contributions}

SK prepared the manuscript. SK, ES, JB and JS were involved in the interpretation of the clinical and pharmacological data. ES, JB, JS, LVB, GVL and BD were involved in study design. $L v B, G V L, B D$ and colleagues conducted the study. FB performed the posthoc ECG analysis. All authors read and approved the final manuscript.

\section{Ethics approval and consent to participate}

All studies were conducted at the Drug Research Unit Ghent in accordance with the Declaration of Helsinki and the Good Clinical Practice guidelines established by the International Conference on Harmonization (ICH). This protocol was approved by the local medical ethics committee (University Hospital of Ghent). All participants have signed informed consent prior to their enrolment.

This trial has been posted on clinicaltrials.gov prior to the conduction of the study (NCT02544217).

\section{Consent for publication}

Not applicable.

\section{Competing interests}

SK has no competing interests. FB is the founding CEO of AMPS. Jan Smeitink is the founding CEO of Khondrion BV.

\section{Publisher's Note}

Springer Nature remains neutral with regard to jurisdictional claims in published maps and institutional affiliations.

\section{Author details}

${ }^{1}$ Radboud Center for Mitochondrial Medicine (RCMM) at the Department of Pediatrics, Radboud university medical center, Geert Grooteplein Zuid 10, PO BOX 9101, 6500, HB, Nijmegen, The Netherlands. ${ }^{2}$ Khondrion BV, Philips van Leydenlaan 15 (427), 6525, EX, Nijmegen, The Netherlands. ${ }^{3}$ Drug Research Unit Ghent, Ghent University Hospital, De Pintelaan 185, 9000 Ghent, Belgium. ${ }^{4}$ Analyzing Medical Parameters for Solutions (AMPS), New York, USA.

Received: 27 June 2017 Accepted: 2 October 2017

Published online: 16 October 2017

\section{References}

1. Calvo SE, Clauser KR, Mootha VK. MitoCarta2.0: an updated inventory of mammalian mitochondrial proteins. Nucleic Acids Res. 2016;44:D1251-7.

2. Koopman WJ, Willems PH, Smeitink JA. Monogenic mitochondrial disorders. N Engl J Med. 2012;366:1132-41.

3. Koene S, Rodenburg RJ, van der Knaap MS, Willemsen MA, Sperl W, Laugel V, Ostergaard E, Tarnopolsky M, Martin MA, Nesbitt V, et al. Natural disease course and genotype-phenotype correlations in complex I deficiency caused by nuclear gene defects: what we learned from 130 cases. J Inherit Metab Dis. 2012;35:737-47.

4. Gorman GS, Schaefer AM, Ng Y, Gomez N, Blakely EL, Alston CL, Feeney C, Horvath R, Yu-Wai-Man P, Chinnery PF, et al. Prevalence of nuclear and mitochondrial DNA mutations related to adult mitochondrial disease. Ann Neurol. 2015;77:753-9.

5. Koopman WJ, Beyrath J, Fung CW, Koene S, Rodenburg RJ, Willems PH, Smeitink JA. Mitochondrial disorders in children: toward development of small-molecule treatment strategies. EMBO Mol Med. 2016;8:311-27.

6. Gorman GS, Chinnery PF, DiMauro S, Hirano M, Koga Y, McFarland R, Suomalainen A, Thorburn DR, Zeviani M, Turnbull DM. Mitochondrial diseases. Nat Rev Dis Primers. 2016;2:16080.

7. Viscomi C. Toward a therapy for mitochondrial disease. Biochem Soc Trans. 2016:44:1483-90.

8. Klopstock T, Yu-Wai-Man P, Dimitriadis K, Rouleau J, Heck S, Bailie M, Atawan A, Chattopadhyay S, Schubert M, Garip A, et al. A randomized placebo-controlled trial of idebenone in Leber's hereditary optic neuropathy. Brain. 2011;134:2677-86.

9. Glover El, Martin J, Maher A, Thornhill RE, Moran GR, Tarnopolsky MA. A randomized trial of coenzyme Q10 in mitochondrial disorders. Muscle Nerve. 2010;42:739-48.

10. Atkuri KR, Cowan TM, Kwan T, Ng A, Herzenberg LA, Herzenberg LA, Enns GM. Inherited disorders affecting mitochondrial function are associated with glutathione deficiency and hypocitrullinemia. Proc Natl Acad Sci U S A. 2009;106:3941-5.

11. Hargreaves IP, Sheena Y, Land JM, Heales SJ. Glutathione deficiency in patients with mitochondrial disease: implications for pathogenesis and treatment. J Inherit Metab Dis. 2005;28:81-8.

12. Wallace DC, Fan W, Procaccio V. Mitochondrial energetics and therapeutics. Annu Rev Pathol. 2010;5:297-348.

13. De Haas R, Das D, Garanto A, Renkema HG, Greupink R, Broek van den P, Pertijs J, Collin RWJ, Willems P, Beyrath J et al. Therapeutic effects of the mitochondrial ROS-redox modulator KH176 in a mammalian model of Leigh Disease. Sci Rep. 2017;7(1):11733

14. Pospischil A, Walther P, Dingemanse J. Phospholipidosis in healthy subjects participating in clinical studies: ultrastructural findings in white blood cells. Exp Toxicol Pathol. 2010;62:567-71.

15. Moore T, Le A, Niemi AK, Kwan T, Cusmano-Ozog K, Enns GM, Cowan TM. A new LC-MS/MS method for the clinical determination of reduced and oxidized glutathione from whole blood. J Chromatogr B Analyt Technol Biomed Life Sci. 2013;929:51-5.

16. Badilini F, Sarapa N. Implications of methodological differences in digital electrocardiogram interval measurement. J Electrocardiol. 2006;39:S152-6. 
17. Badilini F, Vaglio M, Dubois R, Roussel P, Sarapa N, Denjoy I, Extramiana F, Maison-Blanche P. Automatic analysis of cardiac repolarization morphology using Gaussian mesa function modeling. J Electrocardiol. 2008;41:588-94.

18. Johannesen L, Vicente J, Mason JW, Sanabria C, Waite-Labott K, Hong M, Guo P, Lin J, Sorensen JS, Galeotti L, et al. Differentiating drug-induced multichannel block on the electrocardiogram: randomized study of dofetilide, quinidine, ranolazine, and verapamil. Clin Pharmacol Ther. 2014;96:549-58.

19. Drew BJ, Ackerman MJ, Funk M, Gibler WB, Kligfield P, Menon V, Philippides GJ, Roden DM, Zareba W, American Heart Association Acute Cardiac Care Committee of the Council on Clinical Cardiology tCoCN, the American College of Cardiology F. Prevention of torsade de pointes in hospital settings: a scientific statement from the American Heart Association and the American College of Cardiology Foundation. Circulation. 2010;121:1047-60.

20. Isbister GK, Page CB. Drug induced QT prolongation: the measurement and assessment of the QT interval in clinical practice. Br J Clin Pharmacol. 2013; 76:48-57.

Submit your next manuscript to BioMed Central and we will help you at every step:

- We accept pre-submission inquiries

- Our selector tool helps you to find the most relevant journal

- We provide round the clock customer support

- Convenient online submission

- Thorough peer review

- Inclusion in PubMed and all major indexing services

- Maximum visibility for your research

Submit your manuscript at www.biomedcentral.com/submit
Biomed Central 